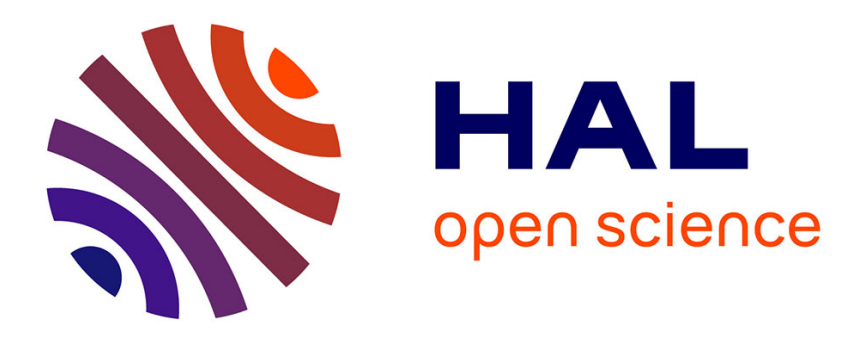

\title{
Conditions of self-oscillations in generalized Persidskii systems
}

Jian Wang, Jésus Mendoza Avila, Denis Efimov, Alexander Yu Aleksandrov, Leonid Fridman

\section{- To cite this version:}

Jian Wang, Jésus Mendoza Avila, Denis Efimov, Alexander Yu Aleksandrov, Leonid Fridman. Conditions of self-oscillations in generalized Persidskii systems. IEEE Transactions on Automatic Control, 2021, 10.1109/TAC.2021.3066581 . hal-03168417

\section{HAL Id: hal-03168417 https://hal.inria.fr/hal-03168417}

Submitted on 13 Mar 2021

HAL is a multi-disciplinary open access archive for the deposit and dissemination of scientific research documents, whether they are published or not. The documents may come from teaching and research institutions in France or abroad, or from public or private research centers.
L'archive ouverte pluridisciplinaire HAL, est destinée au dépôt et à la diffusion de documents scientifiques de niveau recherche, publiés ou non, émanant des établissements d'enseignement et de recherche français ou étrangers, des laboratoires publics ou privés. 


\title{
Conditions of self-oscillations in generalized Persidskii systems
}

\author{
J. Wang, J. Mendoza Avila, D. Efimov, A. Aleksandrov, L. Fridman
}

\begin{abstract}
For a class of generalized Persidskii systems, whose dynamics are described by superposition of a linear part with multiple sector nonlinearities and exogenous perturbations, the conditions of practical stability, instability and oscillatory behavior in the sense of Yakubovich are established. For this purpose the conditions of local instability at the origin and global boundedness of solutions (practical input-to-state stability) are developed in the form of linear matrix inequalities. The proposed theory is applied to investigate robustness to unmodeled dynamics of nonlinear feedback controls in linear systems, and to determine the presence of oscillations in the models of neurons.
\end{abstract}

\section{INTRODUCTION}

Analysis of stability in dynamical systems is a popular and complex problem, whose theory is well-developed nowadays, and it is represented by different notions and approaches [1]. The principal tool to check the stability of an equilibrium or a trajectory is based on the Lyapunov function method [2], [3] and its extensions [4]-[6]. Despite that it has been theoretically proven that existence of an appropriate Lyapunov function is necessary and sufficient for various concepts of stability, there is a barrier for practical application of this procedure: the lack of constructive techniques of finding a needed Lyapunov function for a generic nonlinear system. As a consequence, different canonical forms of nonlinear dynamical models with suitable Lyapunov functions are proposed: Lurie systems, Lipschitz dynamics, homogeneous systems, etc.

The class of Persidskii systems can be considered in such a context. It was first studied in [7] with a linear combination of the integrals of the nonlinearities as a Lyapunov function, and next in [8] the proposed kind of Lyapunov functions was complemented by a combination of the absolute values of the states. This class of models was investigated in the context of diagonal stability [9], neural networks [10], [11], sliding mode controls [12] and digital filters [13]. In a recent work [14], for a kind of generalized Persidskii systems (the right-hand side of the dynamics includes a linear part, several sector nonlinearities and external disturbances) the input-to-

Jian Wang is with Hangzhou Dianzi University, Hangzhou, China.

Denis Efimov is with Inria, Univ. Lille, CNRS, UMR 9189 - CRIStAL, F59000 Lille, France and ITMO University, 49 av. Kronverkskiy, 197101 Saint Petersburg, Russia.

Alexander Yu. Aleksandrov is with Saint Petersburg State University, 7-9 Universitetskaya nab., 199034 Saint Petersburg, Russia.

Jesus Mendoza Avila and Leonid Fridman are with Facultad de Ingeniería, Universidad Nacional Autonoma de México, 04510 Mexico City, Mexico.

This work is partially supported by 111 project No. D17019 (China), by the Ministry of Science and Higher Education of Russian Federation, passport of goszadanie no. 2019-0898, and by the Russian Foundation for Basic Research (grant no. 19-01-00146-a). state stability (ISS) conditions were proposed in the form of linear matrix inequalities (LMIs).

In many cases, the goal mode for a dynamical system is (periodic or chaotic) oscillation [15], [16], which can be entrained by an external force or generated by the system itself (then the latter case corresponds to self- or auto-oscillations). These oscillations can form a desired regime of operation, as in many natural systems [17], [18], or be unwanted parasitic behavior, as chattering in sliding mode controls [19][25], but in all cases the conditions of their persistence are required for analysis and design. That is why this topic attracts many researchers, for examples see [15], [16], [26]-[29] and references therein. A generic and useful theory for studying irregular oscillations was proposed by V.A. Yakubovich [30], [31]. The conditions of oscillations in the sense of Yakubovich are based on existence of two Lyapunov functions [32], [33]. The first Lyapunov function ensures local instability of an equilibrium, while the second one provides attractiveness of a vicinity of that steady state, then under some mild conditions the presence of an oscillating mode can be established.

The aim of this work is to formulate the conditions of existence of oscillations in the sense of Yakubovich for the class of generalized Persidskii systems ${ }^{1}$. To this end, the conditions of local instability at the origin and practical ISS are developed (the latter extends significantly the result obtained in [14]), which can be verified by solving LMIs (a constructive feature that is rarely available for strongly nonlinear systems). The analysis of oscillations is performed following [31], [32]. The proposed conditions are used to investigate robustness of nonlinear feedback applied to linear systems, and when a linear unmodeled dynamics appears in the control channel. Presence of such a dynamics may lead to instability of the system, then appearance of an oscillatory mode (a kind of chattering) implies that a certain closed-loop performance is preserved. Examples of application of the proposed theory to FitzHugh-Nagumo and Hindmarsh-Rose models of neurons are given.

The outline of this paper is as follows. The problem statement is given in Section II. Preliminary results are presented in Section III. Conditions of practical stability, instability and existence of oscillations are established in Section IV. Robustness against unmodeled dynamics is studied in Section V. For illustration of efficiency of the presented conditions, two neural models are considered in Section VI.

\footnotetext{
${ }^{1}$ A preliminary version of this work [34] contains sketched proofs without analysis of neural models.
} 


\section{Notation}

- $\mathbb{R}_{+}=\{x \in \mathbb{R}: x \geq 0\}$, where $\mathbb{R}$ is the set of real numbers.

- $|\cdot|$ and $\|\cdot\|$ denote the absolute value in $\mathbb{R}$ and the Euclidean norm on $\mathbb{R}^{n}$, respectively; for any $\epsilon>0$ define an open ball around the origin by $\mathcal{B}(\epsilon)=\left\{x \in \mathbb{R}^{n}\right.$ : $\|x\|<\epsilon\}$.

- For a (Lebesgue) measurable function $d: \mathbb{R}_{+} \rightarrow \mathbb{R}^{m}$ define the norm $\|d\|_{\left[t_{0}, t_{1}\right)}=$ ess $\sup _{t \in\left[t_{0}, t_{1}\right)}\|d(t)\|$, then $\|d\|_{\infty}=\|d\|_{[0,+\infty)}$ and the set of $d$ with the property $\|d\|_{\infty}<+\infty$ we further denote as $\mathcal{L}_{\infty}^{m}$ (the set of essentially bounded measurable functions).

- A continuous function $\alpha: \mathbb{R}_{+} \rightarrow \mathbb{R}_{+}$belongs to the class $\mathcal{K}$ if $\alpha(0)=0$ and the function is strictly increasing. The function $\alpha: \mathbb{R}_{+} \rightarrow \mathbb{R}_{+}$belongs to the class $\mathcal{K}_{\infty}$ if $\alpha \in \mathcal{K}$ and it is increasing to infinity. A continuous function $\beta: \mathbb{R}_{+} \times \mathbb{R}_{+} \rightarrow \mathbb{R}_{+}$belongs to the class $\mathcal{K} \mathcal{L}$ if $\beta(\cdot, s) \in \mathcal{K}_{\infty}$ and $\beta(s, \cdot)$ is a non-increasing function with $\lim _{t \rightarrow+\infty} \beta(s, t)=0$ for each fixed $s \in \mathbb{R}_{+}$.

- The notation $D V(x) v$ stands for the directional derivative of a continuously differentiable function $V: \mathbb{R}^{n} \rightarrow \mathbb{R}$ evaluated at the point $x$ with respect to a vector $v \in \mathbb{R}^{n}$.

- Denote the identity matrix of dimension $n \times n$ by $I_{n}$, the vector of dimension $n$ with all elements equal 1 by $1_{n}$.

- $\operatorname{diag}\{g\} \in \mathbb{D}^{n}$ represents a diagonal matrix of dimension $n \times n$ with a vector $g \in \mathbb{R}^{n}$ on the main diagonal, where $\mathbb{D}^{n} \subset \mathbb{R}^{n \times n}$ is the set of diagonal matrices. The set of diagonal matrices of dimension $n \times n$ with nonnegative elements will be denoted by $\mathbb{D}_{+}^{n}=\mathbb{D}^{n} \cap \mathbb{R}_{+}^{n \times n}$. For $\Lambda \in$ $\mathbb{D}_{+}^{n}, \Lambda_{i}$ with $i=\overline{1, n}$ corresponds to the $i^{\text {th }}$ element on the main diagonal.

- A series of integers $1,2, \ldots, n$ is denoted by $\overline{1, n}$.

- In work, it is assumed that if the upper limit of a summation or a sequence is smaller than the lower one, then the corresponding terms have to be omitted and the conditions skipped.

\section{PRoblem StATEMENT}

Consider the following class of extended Persidskii systems:

$$
\dot{x}(t)=A_{0} x(t)+\sum_{j=1}^{M} A_{j} f^{j}(x(t))+d(t), t \geq 0,
$$

where $x(t)=\left[x_{1}(t) \ldots x_{n}(t)\right]^{\top} \in \mathbb{R}^{n}$ is the state vector, $x(0) \in \mathbb{R}^{n}$; and $d(t) \in \mathbb{R}^{n}$ is the external perturbation, $d \in \mathcal{L}_{\infty}^{n} ; f^{j}(x)=\left[f_{1}^{j}\left(x_{1}\right) \ldots f_{n}^{j}\left(x_{n}\right)\right]^{\top}, f^{j}(0)=0, j=\overline{1, M}$ are continuous functions (the solutions of the system (1) exist in the forward time at least locally), the matrices $A_{k} \in \mathbb{R}^{n \times n}$ for $k=\overline{0, M}$.

Assumption 1. For any $i=\overline{1, n}, j=\overline{1, M}$ :

$$
s f_{i}^{j}(s)>0 \quad \forall s \in \mathbb{R} \backslash\{0\} .
$$

In the assumption above, it is stated that all nonlinearities belong to a sector and may take zero values at the origin only. Under this hypothesis, after a proper re-indexing and decomposition of $f^{j}$ in (1), there exist $m \in\{0, \ldots, M\}$ and $\mu \in\{m, \ldots, M\}$ such that for all $i=\overline{1, n}$ :

$$
\begin{gathered}
\lim _{s \rightarrow \pm \infty} f_{i}^{z}(s)= \pm \infty, \quad \forall z=\overline{1, m} ; \\
\lim _{s \rightarrow \pm \infty} \int_{0}^{s} f_{i}^{z}(\sigma) d \sigma=+\infty, \quad \forall z=\overline{1, \mu} .
\end{gathered}
$$

Thus, it is supposed that some of the nonlinearities are radially unbounded, and $m=0$ corresponds to the case when all nonlinearities are bounded (at least for negative or positive argument). It is also claimed that other part of these nonlinearities may have unbounded integral (clearly if $m>0$, then all radially unbounded nonlinearities also have unbounded integrals and $\mu \geq m$ due to the introduced sector condition).

If $A_{r}=0$ for all $r=\overline{0, M-1}$ and $\mu=M$, then we recover the system studied by Persidskii in the conventional framework [8], where also was suggested to use a Lyapunov function $V(x)=\sum_{i=1}^{n} \lambda_{i}\left|x_{i}\right|$ with $\lambda_{i}>0$ for all $i=\overline{1, n}$.

Our goal is to propose conditions establishing a presence of oscillating trajectories in (1) (in the sense of Yakubovich, given below in Definition 6).

\section{PRELIMINARIES}

Consider a nonlinear system:

$$
\dot{x}(t)=f(x(t), d(t)), t \geq 0,
$$

where $x(t) \in \mathbb{R}^{n}$ is the state, $d(t) \in \mathbb{R}^{m}$ is the external input, $d \in \mathcal{L}_{\infty}^{m}$, and $f: \mathbb{R}^{n+m} \rightarrow \mathbb{R}^{n}$ is a continuous function, $f(0,0)=0$. For initial condition $x_{0} \in \mathbb{R}^{n}$ and input $d \in \mathcal{L}_{\infty}^{m}$, define corresponding solutions by $x\left(t, x_{0}, d\right)$ for any $t \geq 0$ for which the solutions exist.

\section{A. Basic concepts}

In this work we will be interested in the following stability properties [1], [3], [35]:

Definition 1. The system (2) is called input-to-state practically stable (ISpS), if there are some functions $\beta \in \mathcal{K} \mathcal{L}, \gamma \in \mathcal{K}$ and a constant $c \geq 0$ such that

$$
\left\|x\left(t, x_{0}, d\right)\right\| \leq \beta\left(\left\|x_{0}\right\|, t\right)+\gamma\left(\|d\|_{[0, t)}\right)+c \quad \forall t \geq 0
$$

for any input $d \in \mathcal{L}_{\infty}^{m}$ and any $x_{0} \in \mathbb{R}^{n}$. The function $\gamma$ is called the nonlinear asymptotic gain.

The system is called ISS if $c=0$.

Definition 2. The system (2) is called globally practically stable if there are $\sigma, \gamma \in \mathcal{K}$ and $c \geq 0$ such that

$$
\left\|x\left(t, x_{0}, d\right)\right\| \leq \sigma\left(\left\|x_{0}\right\|\right)+\gamma\left(\|d\|_{[0, t)}\right)+c \quad \forall t \geq 0
$$

for any $x_{0} \in \mathbb{R}^{n}$ and $d \in \mathcal{L}_{\infty}^{m}$. For $d=0$, (2) is called Lagrange stable, and it is called just stable if $c=0$.

It is straightforward to conclude that any ISpS system (2) is globally practically stable with $\sigma(s)=\beta(s, 0)$.

Definition 3. The system (2) is called unstable at the origin for $d=0$, if there is an $\epsilon>0$ such that for any $\delta>0$, $x\left(t^{\prime}, x_{0}, 0\right) \notin \mathcal{B}(\epsilon)$ for some $x_{0} \in \mathcal{B}(\delta)$ and some $t^{\prime} \geq 0$. 
These properties have the following Lyapunov/Chetaev function characterizations:

Definition 4. A smooth function $V: \mathbb{R}^{n} \rightarrow \mathbb{R}_{+}$is called ISpS-Lyapunov function for the system (2) if there exist $\alpha_{1}, \alpha_{2}, \alpha_{3} \in \mathcal{K}_{\infty}, \theta \in \mathcal{K}$ and $r \geq 0$ such that for all $x \in \mathbb{R}^{n}$ and all $d \in \mathbb{R}^{m}$ :

$$
\begin{aligned}
\alpha_{1}(\|x\|) & \leq V(x) \leq \alpha_{2}(\|x\|), \\
D V(x) f(x, d) & \leq r+\theta(\|d\|)-\alpha_{3}(\|x\|) .
\end{aligned}
$$

Such a function $V$ is called ISS-Lyapunov function if $r=0$.

For global practical stability it is enough that an ISpSLyapunov function exists for $\|x\|>X$ with some $X \in \mathbb{R}_{+}$.

Definition 5. A smooth function $V: \mathbb{R}^{n} \rightarrow \mathbb{R}$ is called Chetaev function for the system (2) with $d=0$ if $V(0)=0$, and there exists $\epsilon_{0}>0$ such that $\mathcal{V}^{+} \cap \mathcal{B}(\epsilon) \neq \emptyset$ for any $\epsilon \in\left(0, \epsilon_{0}\right]$, where $\mathcal{V}^{+}=\left\{x \in \mathcal{B}\left(\epsilon_{0}\right): V(x)>0\right\}$, and

$$
D V(x) f(x, 0)>0 \quad \forall x \in \mathcal{V}^{+} .
$$

Theorem 1. [36] The system (2) is ISpS (ISS) if it admits an ISpS (ISS) Lyapunov function.

In the above theorem, existence of the corresponding Lyapunov function is also necessary for ISpS (ISS) property if $f$ is assumed to be locally Lipschitz continuous.

Theorem 2. [37] The system (2) with $d=0$ is unstable if it admits a Chetaev function.

Under additional mild restrictions, existence of a Chetaev function is also necessary for instability [33].

\section{B. Oscillations in the sense of Yakubovich}

A function $h: \mathbb{R}^{n} \rightarrow \mathbb{R}$ is called monotone if the condition $x_{1} \leq x_{1}^{\prime}, \ldots, x_{n} \leq x_{n}^{\prime}$ implies that either $h\left(x_{1}, \ldots, x_{n}\right) \leq$ $h\left(x_{1}^{\prime}, \ldots, x_{n}^{\prime}\right)$ or $h\left(x_{1}, \ldots, x_{n}\right) \geq h\left(x_{1}^{\prime}, \ldots, x_{n}^{\prime}\right)$.

Definition 6. [30], [32] For $-\infty<\pi^{-}<\pi^{+}<+\infty$ a solution $x\left(t, x_{0}, 0\right)$ of the system (2) with $x_{0} \in \mathbb{R}^{n}$ and $d=0$ is called $\left[\pi^{-}, \pi^{+}\right]$-oscillation with respect to an output $h(x)$ (where $h: \mathbb{R}^{n} \rightarrow \mathbb{R}$ is a continuous monotone function) if the solution is defined for all $t \geq 0$ and

$$
\liminf _{t \rightarrow+\infty} h\left(x\left(t, x_{0}, 0\right)\right)=\pi^{-}, \limsup _{t \rightarrow+\infty} h\left(x\left(t, x_{0}, 0\right)\right)=\pi^{+} .
$$

A solution $x\left(t, x_{0}, 0\right)$ of the system (2) is called oscillating, if there exist some output $h$ and constants $\pi^{-}, \pi^{+}$such that the solution $x\left(t, x_{0}, 0\right)$ is $\left[\pi^{-}, \pi^{+}\right]$-oscillation with respect to the output $h$. A forward complete system (2) with $d=0$ is called oscillatory, if for almost all $x_{0} \in \mathbb{R}^{n}$ the solutions $x\left(t, x_{0}, 0\right)$ of the system are oscillating. The oscillatory system (2) is called uniformly oscillatory, if for almost all $x_{0} \in \mathbb{R}^{n}$ for the corresponding solutions $x\left(t, x_{0}, 0\right)$ there exist an output $h$ and constants $\pi^{-}, \pi^{+}$independently on initial conditions.

In other words, the solution $x\left(t, x_{0}, 0\right)$ is oscillating if the output $h\left(x\left(t, x_{0}, 0\right)\right)$ is asymptotically bounded and there is no single limit value of $h\left(x\left(t, x_{0}, 0\right)\right)$ for $t \rightarrow+\infty$. Note that the term "almost all solutions" is used to emphasize that generally the system (2) has a nonempty set of equilibria. The concept of oscillations in the sense of Yakubovich is rather generic, it includes periodical oscillations (limit cycles), quasi-periodical, recurrent and chaotic trajectories.

Despite its complexity, the notion given in Definition 6 has a simple Lyapunov characterization.

Theorem 3. [32] Let the system (2) have two continuously differentiable Lyapunov functions $V_{1}: \mathbb{R}^{n} \rightarrow \mathbb{R}_{+}$and $V_{2}$ : $\mathbb{R}^{n} \rightarrow \mathbb{R}_{+}$such that for $v_{1}, v_{2}, v_{3}, v_{4} \in \mathcal{K}_{\infty}$ the following inequalities are satisfied for all $x \in \mathbb{R}^{n}$ :

$v_{1}(\|x\|) \leq V_{1}(x) \leq v_{2}(\|x\|), v_{3}(\|x\|) \leq V_{2}(x) \leq v_{4}(\|x\|)$,

and for some $0<X_{1}<v_{1}^{-1} \circ v_{2} \circ v_{3}^{-1} \circ v_{4}\left(X_{2}\right)<+\infty$ :

$D V_{1}(x) f(x, 0)>0$ for all $0<\|x\|<X_{1}$ and $x \notin \Pi$;

$D V_{2}(x) f(x, 0)<0$ for all $\|x\|>X_{2}$ and $x \notin \Pi$,

where $\Pi \subset \mathbb{R}^{n}$ is a set with zero Lebesgue measure containing all equilibria of the system, and $\Omega \cap \Pi=\emptyset$ for

$$
\Omega=\left\{x \in \mathbb{R}^{n}: v_{2}^{-1} \circ v_{1}\left(X_{1}\right) \leq\|x\| \leq v_{3}^{-1} \circ v_{4}\left(X_{2}\right)\right\} .
$$

Then the system (2) with $d=0$ is oscillatory.

A Lyapunov function for the linearized system (2) at the origin is a candidate for $V_{1}$ (it can be replaced with Chetaev one [33], as in this work). Instead of existence of the function $V_{2}$ one can require global practical stability of the system (2). In [32], for a class of uniformly oscillatory systems (2) it is shown that these conditions are also necessary.

\section{Stability of extended Persidskii systems}

Stability conditions for the system (1) can be expressed in the form of LMIs:

Theorem 4. [14] Let Assumption 1 be satisfied and there exist $P=P^{\top} \in \mathbb{R}^{n \times n}, \Xi^{k} \in \mathbb{D}_{+}^{n}$ for $k=\overline{0, M}, \Lambda^{j} \in \mathbb{D}_{+}^{n}$ for $j=\overline{1, M}, \Upsilon_{s, j} \in \mathbb{D}_{+}^{n}$ for $s=\overline{0, M-1}$ and $j=\overline{s+1, M}$, $\Gamma=\Gamma^{\top} \in \mathbb{R}^{n \times n}$ such that

$$
\begin{gathered}
P>0 \text { or } P \geq 0, \sum_{z=1}^{\mu} \Lambda^{z}>0 ; \Gamma>0 ; Q \leq 0 ; \\
\sum_{k=0}^{m} \Xi^{k}+2 \sum_{s=0}^{m} \sum_{j=s+1}^{m} \Upsilon_{s, j}>0,
\end{gathered}
$$

where

$$
\begin{gathered}
Q=\left[\begin{array}{cccccc}
Q_{1,1} & Q_{1,2} & Q_{1,3} & \cdots & Q_{1, M+1} & P \\
Q_{1,2}^{\top} & Q_{2,2} & Q_{2,3} & \cdots & Q_{2, M+1} & \Lambda^{1} \\
Q_{1,3}^{\top} & Q_{2,3}^{\top} & Q_{3,3} & \cdots & Q_{3, M+1} & \Lambda^{2} \\
\vdots & \vdots & \vdots & \ddots & \vdots & \vdots \\
Q_{1, M+1}^{\top} & Q_{2, M+1}^{\top} & Q_{3, M+1}^{\top} & \cdots & Q_{M+1, M+1} & \Lambda^{M} \\
P & \Lambda^{1} & \Lambda^{2} & \cdots & \Lambda^{M} & -\Gamma
\end{array}\right] \\
Q_{1,1}=A_{0}^{\top} P+P A_{0}+\Xi^{0} ; Q_{j+1, j+1}=A_{j}^{\top} \Lambda^{j}+\Lambda^{j} A_{j}+\Xi^{j}, j=\overline{1, M} ; \\
Q_{1, j+1}=P A_{j}+A_{0}^{\top} \Lambda^{j}+\Upsilon_{0, j}, j=\overline{1, M} ; \\
Q_{s+1, j+1}=A_{s}^{\top} \Lambda^{j}+\Lambda^{s} A_{j}+\Upsilon_{s, j}, s=\overline{1, M-1}, j=\overline{s+1, M .} .
\end{gathered}
$$

Then the system (1) is ISS.

The proof is based on the following Lyapunov function (recall that $\Lambda_{i}^{j}$ is the $i^{\text {th }}$ element on the main diagonal of $\Lambda^{j}$ ):

$$
V(x)=x^{\top} P x+2 \sum_{j=1}^{M} \sum_{i=1}^{n} \Lambda_{i}^{j} \int_{0}^{x_{i}} f_{i}^{j}(s) d s,
$$


whose time derivative with respect to (1) admits an estimate for $Q \leq 0$ :

$$
\begin{gathered}
D V(x) f(x, d) \leq-x^{\top} \Xi^{0} x-\sum_{j=1}^{M} f^{j}(x)^{\top} \Xi^{j} f^{j}(x) \\
-2 \sum_{j=1}^{M} x^{\top} \Upsilon_{0, j} f^{j}(x)-2 \sum_{s=1}^{M-1} \sum_{j=s+1}^{M} f^{s}(x)^{\top} \Upsilon_{s, j} f^{j}(x) \\
+d^{\top} \Gamma d .
\end{gathered}
$$

The feature of the LMIs (3) consists of a fine treatment of all diagonal items in the off-diagonal blocks of $Q$ represented by the matrices $\Upsilon_{s, j} \geq 0$ for $s=\overline{0, M-1}$ and $j=\overline{s+1, M}$. Without introduction of $\Upsilon_{s, j}$ all corresponding elements would be considered as perturbations, but actually due to the sector condition imposed on nonlinearities $f^{j}(x)$ in Assumption 1, these terms can be sign-definite and, hence, helpful.

Remark 1. The condition $\Xi^{0}>0$ of Theorem 4 for the case $m=0$ can be relaxed to $\Xi^{0}+2 \sum_{j=1}^{K} \Upsilon_{0, j}>0$ under hypothesis that the functions $x_{i} f_{i}^{j}\left(x_{i}\right)$ are radially unbounded for all $i=\overline{1, n}$ and $j=\overline{1, K}$ with some $1 \leq K \leq M$.

If $M=1$, then the system (1) approaches the Lurie form, and the related ISS analysis can be found in [38], [39] (in the latter work a Lyapunov function similar to (4) is used).

\section{CONDITIONS OF STABILITY, INSTABILITY AND OSCILLATIONS}

In this section, first, the conditions of ISpS will be presented for (1) extending the result of Theorem 4 by using Theorem 1 and the Lyapunov function (4) (we will use these results to demonstrate global boundedness). Second, the conditions of instability of (1) with $d=0$ at the origin will be given based on Theorem 2. Finally, the conditions of oscillatory behavior will be proposed combining the previously obtained results in the spirit of Theorem 3 .

\section{A. Global practical stability}

To establish global practical stability property we can use the fact that it is related with ISS, whose conditions for (1) are given in Theorem 4. Note that for this property (since it is simpler than ISS) the derivative of the Lyapunov function (4) should be negative definite in $x$ only for sufficiently big values of the state norm, and even its positive definiteness has to be ensured outside of a ball. These observations provide an insight how we are going to develop the result of Theorem 4, but before we need to introduce additional conventions on the shape and ordering of the functions $f^{j}(x), j=\overline{1, M}$ :

Assumption 2. Let $\limsup _{s \rightarrow \pm \infty} \frac{\left|f_{i}^{j}(s)\right|}{|s|} \in\{0,+\infty\}$ and $\limsup _{s \rightarrow \pm \infty} \frac{\left|f_{i}^{j}(s)\right|}{\left|f_{i}^{k}(s)\right|} \in\{0,+\infty\}$ for all $i=\overline{1, n}$ and all $j \neq k=\overline{1, M}$.

The last condition implies that all $f_{i}^{j}(x), j=\overline{1, M}$ have nonlinear and different asymptotic growth (this property also can be assured by putting the linear components of $f^{j}(x)$ in the linear part explicitly, the same for the functions of a common rate). Roughly speaking, we ask for some growth "uniqueness" of the nonlinearities $f^{j}(x), j=\overline{1, M}$.

Assumption 3. For any $i=\overline{1, n}$ there exists $Z_{i}>0$ such that for all $|s| \geq Z_{i}$ :

$$
\begin{gathered}
s f_{i}^{j}(s)>0, \forall j=\overline{1, M} \\
\left|f_{i}^{1}(s)\right| \geq\left|f_{i}^{2}(s)\right| \geq \cdots \geq\left|f_{i}^{R_{i}}(s)\right| \geq|s| \\
\geq\left|f_{i}^{R_{i}+1}(s)\right| \geq \cdots \geq\left|f_{i}^{M}(s)\right|,
\end{gathered}
$$

where $R_{i} \in\{0, \ldots, M\}$ is an index.

In the above inequalities, the limit terms $f_{i}^{0}$ and $f_{i}^{M+1}$ in the cases $R_{i}=0$ and $R_{i}=M$, respectively, have to be omitted. Under Assumption 2, any weighting these nonlinearities preserves the relations given in Assumption 3 with different $Z_{i}$. These hypotheses are not restrictive, and in the most cases it can be respected by rearranging and reordering the nonlinearities in (1). For brevity of formulation of the result below define $R=\operatorname{sign}\left(\min _{1 \leq i \leq n} R_{i}\right)$ (we have the property $0 \leq R \leq m \leq \mu$, where the indices $m$ and $\mu$ save their meaning under Assumption 3).

Theorem 5. Let assumptions 2, 3 be satisfied and there exist indices $\kappa \in\{1, \ldots, \mu\}$ and $\iota \in\{R, \ldots, m\}$, the matrices $P=P^{\top} \in \mathbb{R}^{n \times n}, \Xi^{k} \in \mathbb{D}^{n}$ for $k=\overline{0, M}, \Lambda^{j} \in \mathbb{D}^{n}$ for $j=\overline{1, M} ; \Upsilon_{s, j} \in \mathbb{D}^{n}$ for $s=\overline{0, M-1}$ and $j=\overline{s+1, M}$, $\Gamma=\Gamma^{\top} \in \mathbb{R}^{n \times n}$ such that

$$
\begin{gathered}
\Lambda^{j} \geq 0, j=\overline{1, \kappa} ; \Xi^{s} \geq 0, \Upsilon_{s, j} \geq 0, s=\overline{0, \iota}, j=\overline{s+1, \iota} ; \\
\Gamma>0 ;\left\{\begin{array}{ll}
P>0 & \mu=0 \\
P \geq 0, \sum_{z=1}^{\kappa} \Lambda^{z}>0 & \mu \geq 1
\end{array} ;\right. \\
\sum_{s=0}^{\iota} \Xi^{s}+2 \sum_{s=0}^{\iota} \sum_{j=s+1}^{\iota} \Upsilon_{s, j}>0 ; Q \leq 0,
\end{gathered}
$$

where the matrix $Q$ is given in Theorem 4. Then the system (1) is globally practically stable.

If, additionally, Assumption 1 holds and $\Lambda^{j} \geq 0, j=$ $\overline{\kappa+1, M}$, then the system (1) is ISpS.

The same claim can be formulated without the requirement on diagonal structure of $\Upsilon_{s, j}$ for $s=\overline{R, \iota}, j=\overline{\iota+1, M}$ and $s=\overline{\iota+1, M-1}, j=\overline{s+1, M}$ or $\Xi^{s}$ for $s=\overline{\iota+1, M}$, but at the price of a more complex writing, which is skipped.

Proof. Consider the Lyapunov function given in (4). Let us show that outside a ball around the origin, it is positive and radially unbounded, and its derivative with respect to (1) admits an estimate given in (5) that is negative and radially unbounded with respect to the state $x$, positive definite with respect to $d$ and has a constant bias (as in Theorem 1).

To prove positivity of $V$ for sufficiently big values of $\|x\|$, first, let us suppose that $\mu=0$ (hence, $R=0$ ), then the term $x^{\top} P x$ contains the elements with the maximal amplitude and $P$ should be positive definite (due to Assumption 2 any weighting the nonlinearities, even with negative multipliers, cannot outperform the quadratic growth in this case). Let $R=$ 0 and $\mu>0$, then the matrix $P$ can be nonnegative definite provided that the first $\kappa$ biggest nonlinearities, which have unbounded integrals, form a positive definite function (that is 
possible for $\mu>0$, and if $\mu=0$ then the condition $P>0$ becomes obligatory). In such a case there exists

$$
Z>\max _{1 \leq i \leq n} Z_{i}
$$

such that $V(x)$ takes only positive values and it is radially unbounded for all $\|x\| \geq Z$ (i.e., the terms with the maximal growth dominate the others for sufficiently big values of $\|x\|$ ). For $R=1$, Assumption 3 implies that in this case $\mu \geq m \geq 1$. Then, either $\kappa \leq \min _{1 \leq i \leq n} R_{i}$ and $\sum_{z=1}^{\kappa} \Lambda^{z}$ has to be positive definite (in such a case the matrix $P$ may be arbitrary), or $P$ has to be nonnegative definite. Again, positivity and radial unboundedness of $V(x)$ for $\|x\| \geq Z$ is recovered as desired. The conditions given in the formulation of the theorem cover all these scenarios. Note that $V(x)$ will be globally positive definite if Assumption 1 is satisfied and $\Lambda^{j} \geq 0$ for all $j=\overline{1, M}$.

To prove negative definiteness of $\dot{V}$ we will use similar arguments. The matrices $\Xi^{s}$ and $\Upsilon_{s, j}$ for $s=\overline{0, \iota}$ and $j=$ $\overline{s+1, \iota}$ in (5) represent the terms with biggest norm for $\|x\| \geq$ $Z$, where $Z$ is a sufficiently big constant as before. According to conditions of the theorem they form a negative definite quadratic form with respect to $f^{s}(x)$ and $x$, and this form will dominate the rest items (the influence of them can be upper bounded by a bias $q>0$ for $\|x\|<Z$, and for $\|x\| \geq Z$ they are covered by the first $\iota$ elements). For $R=0$ the parameter $m$ can take any nonnegative value, and if $m=0$ (hence, $\iota=0$ ) the introduced condition leads to $\Xi^{0}>0$.

The obtained properties are sufficient for global practical stability (see Definition 2), and if additionally Assumption 1 is validated, $\Lambda^{j} \geq 0$ for $j=\overline{1, M}$ and the function $V$ is positive definite, then $V$ is an ISpS-Lyapunov function, and due to Theorem 1 the system (1) is ISpS.

The imposed assumptions, 1, 2 and 3, introduce a rather complex hierarchy for the nonlinearities of the system (1). This is explained by the fact that the considered class of dynamical systems in (1), and the stability problem itself, are rather generic and admit plenty of extensions or modifications.

Remark 2. One such a development can be obtained by observing that the class of Persidskii systems (1) is invariant under a proper linear shift of the nonlinearities. Indeed, let for any $j=\overline{1, M}$ there exist $\vartheta_{j} \in \mathbb{R}$ such that

$$
s \tilde{f}_{i}^{j}(s)>0 \quad \forall s \in \mathbb{R} \backslash\{0\}
$$

for all $i=\overline{1, n}$, where $\tilde{f}^{j}(x)=f^{j}(x)-\vartheta_{j} x$, then Assumption 3 is verified for $\tilde{f}^{j}(x)$. Thus, a properly introduced auxiliary linear feedback does not change the sector property of the system nonlinearities, then the following equivalent dynamics can be studied:

$$
\dot{x}(t)=\tilde{A}_{0} x(t)+\sum_{j=1}^{M} A_{j} \tilde{f}^{j}(x(t))+d(t), t \geq 0,
$$

where $\tilde{A}_{0}=A_{0}+\sum_{j=1}^{M} \vartheta_{j} A_{j}$. Therefore, if for this new system the conditions of Theorem 5 are satisfied, then (1) possesses the global practical stability property (or ISpS). The advantage of such a transformation is that the matrix $\tilde{A}_{0}$ may have an improved stability characteristic (see the next section).
Remark 3. If Assumption 2 fails to satisfy, then the LMIs of Theorem 5 should be complemented by additional restrictions to guarantee that the meaningful terms with the same growth have a negative definite sum.

The results of this subsection introduce a series of tools for analysis of boundedness of trajectories in the extended Persidskii system (1), these tools can be combined and also relaxed considering particular scenarios and applications.

\section{B. Local instability at the origin}

For local instability, it is sufficient to ensure positivity of $V$ at some vicinity of the origin and positive definiteness of the derivative $\dot{V}$ in the domain where $V(x)>0$. To this end, let us introduce a reordering of the nonlinearities:

Assumption 4. Let $\limsup _{s \rightarrow 0} \frac{\left|f_{i}^{j}(s)\right|}{|s|} \in\{0,+\infty\}$ and $\limsup _{s \rightarrow 0} \frac{\left|f_{i}^{j}(s)\right|}{\left|f_{i}^{k}(s)\right|} \in\{0,+\infty\}$ for all $i=\overline{1, n}$ and all $j \neq k=\overline{1, M}$.

This condition reads that all $f_{i}^{j}(x), j=\overline{1, M}$ have nonlinear and different local growth at the origin.

Assumption 5. There exist $Z>0, \eta>0$ such that for all $s \in(-Z, 0) \cup(0, Z)$ :

$$
\begin{gathered}
\eta s f_{i}^{j}(s) \geq \int_{0}^{s} f_{i}^{j}(\sigma) d \sigma>0, \forall j=\overline{1, M} \\
\left|f_{i}^{1}(s)\right| \geq\left|f_{i}^{2}(s)\right| \geq \cdots \geq\left|f_{i}^{R_{i}}(s)\right| \geq|s| \\
\geq\left|f_{i}^{R_{i}+1}(s)\right| \geq \cdots \geq\left|f_{i}^{M}(s)\right|
\end{gathered}
$$

where $R_{i} \in\{0, \ldots, M\}$ is an index for all $i=\overline{1, n}$.

As we can see, all restrictions are imposed for $\|x\|<Z$ and there is no requirement on radial unboundedness of functions and integrals for a local investigation.

Theorem 6. Let assumptions 4, 5 hold and there are indices $\kappa \in\{1, \ldots, M\}$ and $\iota \in\{1, \ldots, n\}$, the matrices $P=P^{\top} \in$ $\mathbb{R}^{n \times n} ; \Xi^{k} \in \mathbb{D}^{n}$ for $k=\overline{0, M} ; \Lambda^{j} \in \mathbb{D}^{n}$ for $j=\overline{1, M}$; $\Upsilon_{s, j} \in \mathbb{D}^{n}$ for $s=\overline{0, M-1}$ and $j=\overline{s+1, M}$ such that

$$
\begin{aligned}
& \Lambda_{\iota}^{z} \geq 0, z=\overline{1, \kappa} ;\left\{\begin{array}{ll}
\sum_{z=1}^{\kappa} \Lambda_{\iota}^{z}>0 & \kappa \leq R_{\iota} \\
P_{\iota, \iota} \geq 0, P_{\iota, \iota}+\sum_{z=1}^{\kappa} \Lambda_{\iota}^{z}>0 & \kappa>R_{\iota}
\end{array} ;\right. \\
& \Xi^{0}+\rho P \leq 0, \Xi^{j} \leq 0, \Upsilon_{0, j}+\rho \eta \Lambda^{j} \leq 0, j=\overline{1, M}, \\
& \Upsilon_{s, j} \leq 0, s=\overline{1, M-1}, j=\overline{s+1, M} ; Q \geq 0,
\end{aligned}
$$

where $\rho>0$ is a parameter and the matrix $Q$ is given in Theorem 4. Then (1) with $d=0$ is unstable at the origin.

Proof. Consider again the function $V(x)$ given in (4) and its derivative with respect to (1) for the case $d=0$, which admits a lower estimate for $Q \geq 0$ :

$$
\begin{gathered}
D V(x) f(x, 0) \geq-x^{\top} \Xi^{0} x-\sum_{j=1}^{M} f^{j}(x)^{\top} \Xi^{j} f^{j}(x) \\
-2 \sum_{j=1}^{M} x^{\top} \Upsilon_{0, j} f^{j}(x)-2 \sum_{s=1}^{M-1} \sum_{j=s+1}^{M} f^{s}(x)^{\top} \Upsilon_{s, j} f^{j}(x) .
\end{gathered}
$$


Let us check the conditions of Theorem 2 showing that $V$ is a Chetaev function for (1) under the hypotheses of the theorem.

It is necessary to ensure that at least for one coordinate, say $x_{\iota}$ with an index $\iota \in\{1, \ldots, n\}$, when all the coordinates $x_{i}=0$ for $i=\overline{1, n}$ and $i \neq \iota$, the function $V(x)$ takes positive values. Indeed, if it is the case, then even for nonzero $x_{i}$ with $i \neq \iota$ it will be a small neighborhood around the origin containing the axis $x_{\iota}$ with $V(x)>0$. According to Assumption 5, the nonlinearities are sorted in the decreasing order, and if $\kappa \leq R_{\iota}$, then the first $\kappa$ nonlinearities are bigger than the linear part and other terms, $\Lambda_{\iota}^{z} \geq 0$ for $z=\overline{1, \kappa}$ and $\sum_{z=1}^{\kappa} \Lambda_{\iota}^{z}>0$. Hence, $V(x)$ is positive definite in $x_{\iota}$ (we have to recall here assumptions 4 and 5). If $\kappa>R_{\iota}$, then in addition it is necessary to ensure nonnegative definiteness of $P_{\iota, \iota} x_{\iota}^{2}$, and we conclude on the same property of $V$.

Finally, let us check that $D V(x) f(x, 0)$ is locally positive definite in the domain where $V(x)>0$. Under the conditions of theorem, $D V(x) f(x, 0) \geq \rho V(x)$ that clearly implies the desired conclusion. The result follows by Theorem 2 .

As previously for Theorem 5, the formulation of Theorem 6 admits various relaxations that are omitted for brevity (for example, the requirement on diagonal structure of $\Upsilon_{s, j}$ for $s=$ $\overline{R, \kappa}, j=\overline{\kappa+1, M}$ and $s=\overline{\kappa+1, M-1}, j=\overline{s+1, M}$, or $\Xi^{s}$ for $s=\overline{\kappa+1, M}$ can be skipped).

\section{Existence of oscillations}

Following the result of Theorem 3, it is straightforward to formulate the required conditions:

Theorem 7. Let the requirements of theorems 5, 6 be satisfied and the system (1) with $d=0$ admit the only equilibrium at the origin. Then (1) with $d=0$ is oscillatory, and for any $d \in \mathcal{L}_{\infty}^{n}$ the solutions stay bounded.

Proof. Under these restrictions, the set $\Pi$ from Theorem 3 contains just the origin. Fulfillment of the conditions of Theorem 5 ensures the global practical stability (it replaces existence of the function $V_{2}$ from Theorem 3), while Theorem 6 guarantees instability of the only steady state (it substitutes existence of the function $V_{1}$ from Theorem 3 ). Hence, there is a compact forward invariant set $\Omega \subset \mathbb{R}^{n}$ attracting asymptotically almost all trajectories of the system, and obviously $\Omega \cap \Pi=\emptyset$. Then similarly to the proof of Theorem 3, there is a coordinate $x_{i}$ with $i \in\{1, \ldots, n\}$ such that these trajectories are oscillating for $h(x)=x_{i}$.

Remark 4. It is clear that the LMIs given in theorems 5 and 6 are of the same kind, then it can be difficult to resolve them simultaneously. A way to fix this issue is to apply the linear shifts as in Remark 2 by checking, for example, the conditions of Theorem 6 for $\tilde{f}^{j}(x), j=\overline{1, M}$ and $\tilde{A}_{0}$.

Let us demonstrate the efficiency of the developed theory in applications.

\section{ROBUSTNESS AGAINST UNMODELED DYNAMICS}

Consider a linear system:

$$
\dot{z}(t)=A z(t)+B u(t)
$$

where $z(t) \in \mathbb{R}^{\nu}$ and $u(t) \in \mathbb{R}^{\varrho}$ are the state and the control vectors, $\nu>0$ and $\varrho>0$ are some integers, $A \in \mathbb{R}^{\nu \times \nu}$ and $B \in \mathbb{R}^{\nu \times \varrho}$. Assume that there is a control input

$$
u(t)=-\sum_{j=1}^{M} K_{j} \phi^{j}(z(t))
$$

where $M>0$ is an integer and $\phi^{j}: \mathbb{R}^{\nu} \rightarrow \mathbb{R}^{\nu}$ are secor nonlinear functions, $\phi^{j}(z)=\left[\phi_{1}^{j}\left(z_{1}\right) \ldots \phi_{\nu}^{j}\left(z_{\nu}\right)\right]^{\top}, \phi^{j}(0)=0$, $j=\overline{1, M} ; K_{j} \in \mathbb{R}^{\varrho \times \nu}$ for $j=\overline{1, M}$; and the closedloop system is stable and possesses a desired regulation performance (clearly it is a system in the form (1) with $d=0$ ). Next, assume that in the control channel there is an unmodeled dynamics (actuator) represented by a linear stable filter:

$$
\begin{gathered}
u(t)=C \zeta(t), v(t)=-\sum_{j=1}^{M} K_{j} \phi^{j}(z(t)), \\
\dot{\zeta}(t)=D \zeta(t)+G v(t)
\end{gathered}
$$

where $\zeta(t) \in \mathbb{R}^{\varpi}$ is the state of the filter for an integer $\varpi>0$, $v(t) \in \mathbb{R}^{\varrho}$ is the control applied to the actuator but designed for the system (6), and $u(t) \in \mathbb{R}^{\varrho}$ is the output, which is the control for the system (6); $C \in \mathbb{R}^{\varrho \times \varpi}, D \in \mathbb{R}^{\varpi \times \varpi}$ and $G \in \mathbb{R}^{\varpi \times \varrho}$. The closed-loop dynamics of (6) and (7) can be rewritten in the form of (1) with $d=0$ :

$$
\begin{gathered}
x=\left[z_{1}, \ldots, z_{\nu}, \zeta_{1}, \ldots, \zeta_{\varpi}\right]^{\top} \in \mathbb{R}^{n}, n=\nu+\varpi \\
f^{j}(x)=\left[\phi_{1}^{j}\left(x_{1}\right), \ldots, \phi_{\nu}^{j}\left(x_{\nu}\right), x_{\nu+1}, \ldots, x_{n}\right]^{\top}, j=\overline{1, M}, \\
A_{0}=\left[\begin{array}{cc}
A & B C \\
0 & D
\end{array}\right], A_{j}=-\left[\begin{array}{c}
0 \\
G
\end{array}\right]\left[\begin{array}{cc}
K_{j} & 0
\end{array}\right], j=\overline{1, M} .
\end{gathered}
$$

Therefore, using the result of Theorem 7 we can verify the oscillatory property of the closed-loop system under such an unmodeled actuator.

The previous problem statement is a well-studied case in the sliding mode control theory, where the observed oscillations are called "chattering" [19]-[21], [23]-[25].

Example 1. Let $\nu=\varrho=1, \varpi=2$ and

$$
\begin{gathered}
A=0, B=1, C=\left[\begin{array}{ll}
1 & 0
\end{array}\right], D=\left[\begin{array}{cc}
-\delta & \delta \\
0 & -\delta
\end{array}\right], \\
G=\left[\begin{array}{l}
0 \\
\delta
\end{array}\right], M=1, \phi^{1}(z)=|z|^{\alpha} \operatorname{sign}(z),
\end{gathered}
$$

where $\alpha \in(0,1)$ and $\delta>0$ are tuning parameters, $K_{1}>0$. Such a control is used for finite-time stabilization [40]. Hence,

$$
A_{0}=\left[\begin{array}{ccc}
0 & 1 & 0 \\
0 & -\delta & \delta \\
0 & 0 & -\delta
\end{array}\right], A_{1}=\left[\begin{array}{ccc}
0 & 0 & 0 \\
0 & 0 & 0 \\
-\delta K_{1} & 0 & 0
\end{array}\right] \text {, }
$$

and the matrix $A_{0}$ is not Hurwitz, it has one zero eigenvalue and two equal to $-\delta$.

To check the conditions of Theorem 5 select $P=P^{\top}>$ $0, \Lambda^{1}=\operatorname{diag}\left[\Lambda_{1}^{1}, 0,0\right]^{\top} \in \mathbb{D}^{3}$ with $\Lambda_{1}^{1}>0, \Xi^{0}=$ $\operatorname{diag}\left[0, \Xi_{2}^{0}, \Xi_{3}^{0}\right]^{\top} \in \mathbb{D}_{+}^{3}$ with $\Xi_{2}^{0}>0, \Xi_{3}^{0}>0$, and the matrix $\Upsilon_{0,1} \in \mathbb{R}^{3 \times 3}$ in a generic form (full matrix) with requirement

$$
\left(\Upsilon_{0,1}\right)_{1,1}>0
$$




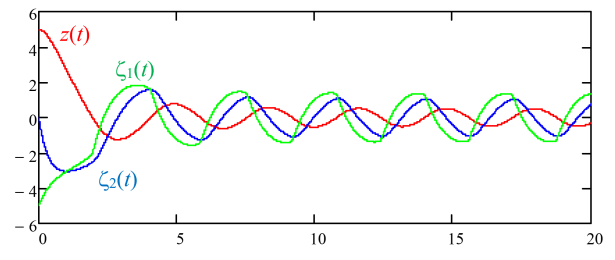

Figure 1. Simulation results of (6), (7) for $\delta=2, K_{1}=2$ and $\alpha=0.25$

i.e., the first element of this matrix corresponding to the term $|z|^{\alpha+1}$ is positive. It is obvious that the Lyapunov function $V(x)$ given in (4) is positive definite under introduced restrictions. It is also straightforward to verify that there exist the matrices as above such that $Q \leq 0$ and in (5)

$$
\begin{gathered}
D V(x) f(x, 0) \leq-\left(\Upsilon_{0,1}\right)_{1,1}|z|^{\alpha+1}-\Xi_{2}^{0} \zeta_{1}^{2} \\
-\Xi_{3}^{0} \zeta_{2}^{2}+2\left[\left|\left(\Upsilon_{0,1}\right)_{2,1}\right|\left|\zeta_{1}\right||z|^{\alpha}+\left|\left(\Upsilon_{0,1}\right)_{3,1}\right|\left|\zeta_{2}\right||z|^{\alpha}\right],
\end{gathered}
$$

then the required global boundedness of trajectories is proven. Indeed, applying Young's inequality for $i=1,2$ we obtain $2\left|\zeta_{i}\right||z|^{\alpha} \leq \varepsilon_{i} \zeta_{i}^{2}+\frac{1}{\varepsilon_{i}}|z|^{2 \alpha}$, then $\varepsilon_{i}>0$ can be selected sufficiently small to satisfy $\varepsilon_{i}\left|\left(\Upsilon_{0,1}\right)_{i+1,1}\right|<\Xi_{i+1}^{0}$, and $|z|^{2 \alpha}$ is radially dominated by $|z|^{\alpha+1}$ for $\alpha \in(0,1)$. Note that in the linear case, i.e., for $\alpha=1$, some additional restrictions on $\delta$ and $K_{1}$ have to be imposed to prove the boundedness.

To use Theorem 6 (see [22] for application of Chetaev function in similar context), take any $\vartheta_{1}>0$ and shift the nonlinearity $f^{1}$ by linear feedback (as in Remark 2 ), then we obtain the new matrix

$$
\tilde{A}_{0}=A_{0}+\vartheta_{1} A_{1}=\left[\begin{array}{ccc}
0 & 1 & 0 \\
0 & -\delta & \delta \\
-\vartheta_{1} \delta K_{1} & 0 & -\delta
\end{array}\right]
$$

It is straightforward to verify that always there exists a value of $\vartheta_{1}$ sufficiently big such that the matrix $\tilde{A}_{0}$ has a positive eigenvalue in the subspace of the variable $z$. Since $\alpha \in(0,1)$, then also for any value of $\vartheta_{1}$ the function $\tilde{f}_{1}^{j}(x)=f_{1}^{j}\left(x_{1}\right)-$ $\vartheta_{1} x_{1}$ stays in the required sector onto a vicinity of the origin. Take $P=P^{\top}>0, \Lambda^{1}=\operatorname{diag}\left[\Lambda_{1}^{1}, 0,0\right]^{\top} \in \mathbb{D}_{+}^{3}$ with $\Lambda_{1}^{1}>0$, $\Xi^{0} \in \mathbb{D}^{3}$ with $\Xi_{1}^{0}<0$, and $\Upsilon_{0,1} \in \mathbb{D}^{3}$ with $\left(\Upsilon_{0,1}\right)_{1,1}<0$, then the conditions of Theorem 6 are satisfied for $\iota=1$.

By Theorem 7 the system is oscillatory. The results of simulation for $\delta=2, K_{1}=2$ and $\alpha=0.25$ are shown in Fig. 1 (in this case $u=\zeta_{1}$ ).

\section{OSCILLATIONS IN NEURON MODELS}

We will consider two models of neural dynamics.

\section{A. FitzHugh-Nagumo model}

This model describes a prototype of an excitable neuron, or a relaxation oscillator [18]:

$$
\begin{aligned}
& \dot{x}_{1}(t)=x_{1}(t)-\frac{1}{3} x_{1}^{3}(t)-x_{2}(t)+I, \\
& \dot{x}_{2}(t)=\frac{1}{\tau}\left(x_{1}(t)-b x_{2}(t)+a\right),
\end{aligned}
$$

where $x_{1}(t) \in \mathbb{R}$ and $x_{2}(t) \in \mathbb{R}$ are the membrane voltage and linear recovery variable, respectively; $I \in \mathbb{R}$ is a constant magnitude of external stimulus current; $a \geq 0$ and $b \geq 0$ are parameters (for $a=b=0$ the system can be reduced to Van der Pol oscillator); $t \geq 0$. If $b \in(0,1)$, then the system admits the only equilibrium with the coordinates $x_{10} \in \mathbb{R}$ and $x_{20} \in \mathbb{R}$ satisfying:

$$
\left(\frac{1}{3} x_{10}^{2}+\frac{1-b}{b}\right) x_{10}=I-\frac{a}{b}, b x_{20}=x_{10}+a,
$$

and for brevity further we assume this constraint. Obviously, this system is in the form (1) with $n=2$ and $M=1$ :

$$
\begin{gathered}
A_{0}=\left[\begin{array}{cc}
1 & -1 \\
\frac{1}{\tau} & -\frac{b}{\tau}
\end{array}\right], A_{1}=\left[\begin{array}{cc}
-\frac{1}{3} & 0 \\
0 & 0
\end{array}\right], \\
f^{1}(x)=\left[\begin{array}{l}
x_{1}^{3} \\
x_{2}^{3}
\end{array}\right], d=\left[\begin{array}{c}
I \\
\frac{a}{\tau}
\end{array}\right] .
\end{gathered}
$$

To check global boundedness of the solutions, Theorem 5 can be used directly with $m=\mu=R=1$, and in particular

$$
V(x)=\frac{1}{2}\left(x_{1}^{2}+\tau x_{2}^{2}\right), \dot{V} \leq \frac{3}{2} x_{1}^{2}-\frac{1}{3} x_{1}^{4}-\frac{b}{2} x_{2}^{2}+\frac{1}{2} I^{2}+\frac{a^{2}}{2 b}
$$

is an admissible choice. To verify local instability at the equilibrium applying Theorem 6 we will shift it to the origin via the change of coordinates $e_{1}(t)=x_{1}(t)-x_{10}, e_{2}(t)=$ $x_{2}(t)-x_{20}$, then this error dynamics will preserve the form of the original system for $d=0$ and

$$
f^{1}(e)=\left[\begin{array}{c}
e_{1}\left(e_{1}^{2}+3 x_{10}\left(e_{1}+x_{10}\right)\right) \\
e_{2}\left(e_{2}^{2}+3 x_{20}\left(e_{2}+x_{20}\right)\right)
\end{array}\right] .
$$

Next, for any value of $x_{10}$ and $x_{20}$, at least in a vicinity of the origin, the sector condition is verified for this $f^{1}(e)$, then $V(e)=e^{\top} P e, P=P^{\top}$ is the required Chetaev function (the Chetaev function for the linearized dynamics) that can satisfy the conditions of Theorem 6. In such a case, by Theorem 7, the system is oscillatory.

\section{B. Hindmarsh-Rose model}

This model of neuronal activity represents well the spikingbursting behavior of the membrane potential observed in vivo, and it is more complicated [18]:

$$
\begin{aligned}
& \dot{x}_{1}(t)=x_{2}(t)-a x_{1}^{3}(t)+b x_{1}^{2}(t)-x_{3}(t)+I, \\
& \dot{x}_{2}(t)=c-k x_{1}^{2}(t)-x_{2}(t), \\
& \dot{x}_{3}(t)=r\left[s\left(x_{1}(t)-x_{R}\right)-x_{3}(t)\right],
\end{aligned}
$$

where again $x_{1}(t) \in \mathbb{R}$ is the membrane potential, and $x_{2}(t), x_{3}(t) \in \mathbb{R}$ represent the transport of ions across the membrane $\left(x_{2}(t)\right.$ stays for sodium and potassium ions, and it is called the spiking variable; $x_{3}(t)$ corresponds to other ions, it is the bursting variable); $I \in \mathbb{R}$ is the stimulus current, $a, b, c, k, r, s, x_{R} \in \mathbb{R}_{+}$are parameters. This system can be also presented in the form (1) with $n=3$ and $M=2$ :

$$
A_{0}=\left[\begin{array}{ccc}
-b \vartheta & 1 & -1 \\
k \vartheta & -1 & 0 \\
r s & 0 & -r
\end{array}\right], A_{1}=\left[\begin{array}{ccc}
-a-b & 0 & 0 \\
k & 0 & 0 \\
0 & 0 & 0
\end{array}\right] \text {, }
$$




$$
\begin{gathered}
A_{2}=\left[\begin{array}{ccc}
b & 0 & 0 \\
-k & 0 & 0 \\
0 & 0 & 0
\end{array}\right], d=\left[\begin{array}{c}
I \\
c \\
-r s x_{R}
\end{array}\right] \\
f^{1}(x)=\left[\begin{array}{l}
x_{1}^{3} \\
x_{2}^{3} \\
x_{3}^{3}
\end{array}\right], f^{2}(x)=\left[\begin{array}{l}
\phi\left(x_{1}\right) \\
\phi\left(x_{2}\right) \\
\phi\left(x_{3}\right)
\end{array}\right], \\
\phi(x)=\left(x^{2}+x+\vartheta\right) x
\end{gathered}
$$

and $\vartheta>0$ is a parameter. Let us verify only global boundedness of the solutions using Theorem 5 (the local instability can be checked by considering linearized dynamics at equilibria). Note that Assumption 2 is not satisfied in this case, then the LMIs of Theorem 5 complemented by restriction given in Remark 3 are feasible with $m=\mu=R=2$ and $\kappa=\iota=1$ for $a=1, b=3, k=0.5, s=4, r=10^{-3}$ and $\vartheta=0.1$.

\section{CONCLUSIONS}

The most existing works on Persidskii systems deal with their standard form (i.e., with $M=1$ in (1)), and the case with $M>1$ was for the first time studied recently in [14] (multiple nonlinearities enlarge the domain of possible applications), where a careful treatment of cross terms between $f^{j}$ and $x$ in the derivative of $V$ is developed in order to obtain non-conservative stability conditions. Following [14], the present work extends those results to practical ISS analysis (in Theorem 5 almost all conditions of Theorem 4 are relaxed) and to instability verification (Theorem 6) using LMIs. The obtained in Theorem 7 conditions of existence of oscillations in the sense of Yakubovich were never proposed for any kind of Persidskii systems. The results are used to investigate robustness to linear unmodeled dynamics of nonlinear controls for linear systems. The efficiency of the obtained conditions is illustrated on models of neurons.

\section{REFERENCES}

[1] H. Khalil, Nonlinear Systems, 3rd ed. Upper Saddle River, New Jersey: Prentice Hall, 2002.

[2] I. Malkin, Theory of Stability of Motion. US Atomic Energy Commission, 1952, vol. Translation Series: Physics and Mathematics.

[3] W. Hahn, Stability of Motion. New York: Springer-Verlag, 1967.

[4] J. LaSalle and S. Lefchetz, Stability by Liapunov's Direct Method with Aplications. New York: Academic Press, 1961.

[5] A. van der Schaft, $L_{2}$-gain and passivity techniques in nonlinear control, ser. Lecture Notes in Control and Information Sciences. London: Springer-Verlag London Ltd., 1996, vol. 218.

[6] Y. Lin, E. D. Sontag, and Y. Wang, "A smooth converse Lyapunov theorem for robust stability," SIAM Journal on Control and Optimization, vol. 34, no. 1, pp. 124-160, 1996.

[7] E. Barbashin, "On construction of Lyapunov functions for nonlinear systems," in Proc. 1st IFAC World Congress, Moscow, 1961, pp. 742 751.

[8] S. Persidskii, "Problem of absolute stability," Automation and Remote Control, vol. 12, pp. 1889-1895, 1969.

[9] E. Kazkurewicz and A. Bhaya, Matrix Diagonal Stability in Systems and Computation. Boston: Birkhauser, 1999.

[10] J. Hopfield and D. Tank, "Computing with neural circuits: a model," Science, vol. 233, pp. 625-633, 1986.

[11] E. Sontag, "Recurrent networks," in Some Topics in Neural Networks and Control, 1993.

[12] A. Aparicio, L. Fridman, and D. Efimov, "Stabilization of systems with switchings on the axis of their coordinates and its input-to-state properties," Nonlinear Analysis: Hybrid Systems, vol. 32, pp. 10-18, 2019.
[13] K. Erickson and A. Michel, "Stability analysis of fixed-point digital filters using computer generated Lyapunov functions - Part I: Direct form and coupled form filters," IEEE Trans. on Circuits and Systems, vol. 32, pp. 113-132, 1985.

[14] D. Efimov and A. Aleksandrov, "Robust stability analysis and implementation of Persidskii systems," in Proc. 58th IEEE Conference on Decision and Control (CDC), Nice, 2019.

[15] A. Fradkov and A. Pogromsky, Introduction to oscillations and chaos. Singapore: World Scientific, 1998.

[16] G. Leonov, I. Burkin, and A. Shepelyavyi, Frequency Methods in Oscillation Theory. Dordrecht: Kluwer, 1995, in Russian: 1992.

[17] A. Goldbeter, "A model for circadian oscillations in the drosophila period protein (per)," Proc. Royal Society of London. Series B: Biological Sciences, vol. 261, no. 1362, 1995.

[18] E. M. Izhikevich, Dynamical Systems in Neuroscience: The Geometry of Excitability and Bursting. Cambridge, MA: The MIT Press, 2007.

[19] Y. Shtessel and L. Young Ju, "New approach to chattering analysis in systems with sliding modes," in Proc. 35th IEEE Conference Decision and Control, Kobe, 1996, pp. 790-795.

[20] F. L., Variable Structure, Sliding Mode and Nonlinear Control. London: Springer Verlag, 1999, ch. The problem of chattering: an averaging approach, pp. 363-386.

[21] G. Bartolini, A. Ferrara, and E. Usai, "Chattering avoidance by second order sliding mode control," IEEE Transactions on Automatic Control, vol. 43, no. 2, pp. 241-246, 1998.

[22] L. Fridman and L. A., Sliding Mode Control in Engineering. New York: Marcel Dekker, 2002, ch. Higher order sliding modes in Sliding Mode Control in Engineering, pp. 53-101.

[23] A. Pisano and E. Usai, "Contact force regulation in wire-actuated pantographsvia variable structure control and frequency-domain techniques," Int. J. Control, vol. 81, no. 11, pp. 1747-1762, 2008.

[24] I. Boiko, Discontinuous Control Systems, Frequency Domain Analysis and Design. Boston, MA: Birkhäuser, 2009.

[25] A. Levant, "Chattering analysis," IEEE Transactions on Automatic Control, vol. 55, no. 6, pp. 1380-1389, 2010.

[26] J. M. Goncalves, A. Megretski, and M. A. Dahleh, "Global stability of relay feedback systems," IEEE Transactions on Automatic Control, vol. 46, no. 4, pp. 550-562, 2001.

[27] S. Martinez, J. Cortes, and F. Bullo, "Analysis and design of oscillatory control systems," IEEE Trans. Autom. Control, vol. 48, no. 7, pp. 11641177, 2003.

[28] F. D. Meglio, G.-O. Kaasa, and N. Petit, "A first principle model for multiphase slugging flow in vertical risers," in Proc. 48th IEEE Conf. on Decision and Control. Shanghai: IEEE, 2009, pp. 8244-8251.

[29] B. Andrievsky, N. Kuznetsov, G. Leonov, and A. Pogromsky, "Hidden oscillations in aircraft flight control system with input saturation," in IFAC Proceedings Volumes, vol. 46, no. 12, 2013, pp. 75-79.

[30] V. Yakubovich, "Frequency oscillations conditions in nonlinear systems with stationary single nonlinearity," Siberian Math. J., vol. 14, no. 2, pp. 265-289, 1973.

[31] V. Yakubovich and E. Tomberg, "Conditions for self-induced oscillations in nonlinear systems," Siberian Math. J., vol. 30, pp. 641-653, 1989.

[32] D. Efimov and A. Fradkov, "Oscillatority of nonlinear systems with static feedback," SIAM J. Control Optimization, vol. 48, no. 2, pp. 618640, 2009.

[33] D. Efimov and W. Perruquetti, "On conditions of oscillations and multihomogeneity," Mathematics of Control, Signals, and Systems, vol. 28, no. 1, pp. 1-37, 2015.

[34] J. Wang, J. Mendoza Avila, D. Efimov, A. Aleksandrov, and L. Fridman, "On existence of oscillations in Persidskii systems," in Proc. 21rst IFAC World Congress, Berlin, 2020.

[35] S. Dashkovskiy, D. Efimov, and E. Sontag, "Input to state stability and allied system properties," Automation and Remote Control, vol. 72, no. 8, pp. 1579-1614, 2011

[36] E. D. Sontag and Y. Wang, "On characterizations of the input-to-state stability property," Systems Control Lett., vol. 24, no. 5, pp. 351-359, 1995.

[37] N. Chetaev, The Stability of Motion. New York: Pergamon Press, 1961, (English translation).

[38] M. Arcak and A. Teel, "Input-to-state stability for a class of Lurie systems," Automatica J. IFAC, vol. 38, no. 11, pp. 1945-1949, 2002.

[39] S. Dashkovskiy, "Practical examples of ISS systems," IFACPapersOnLine, vol. 52, no. 16, pp. 1-6, 2019, 11th IFAC Symposium on Nonlinear Control Systems NOLCOS 2019.

[40] E. Bernuau, D. Efimov, W. Perruquetti, and A. Polyakov, "On homogeneity and its application in sliding mode," J. Franklin Institute, vol. 351 , no. 4, pp. 1866-1901, 2014. 